\title{
A Practical Load-optimized VCO Design for Low-jitter 5V 500 MHz Digital Phase-locked Loop*
}

\author{
CHUA-CHIN WANG ${ }^{\dagger}$, YU-TSUN CHIEN and YING-PEI CHEN \\ Dept. of Electrical Engineering, National Sun Yat-Sen University, Kaohsiung, Taiwan 80424 \\ (Received 5 June 1999; In final form 1 October 1999)

\begin{abstract}
In high-speed digital systems and high-resolution display devices, the jitter effect of phase-locked loops (PLL) limits the system performance. Power supply noise coupling is one of the major causes of PLL jitter problems, especially with mixed-signal systems. The paper presents a targeted $5.0 \mathrm{~V} 500 \mathrm{MHz}$ PLL which is implemented by a $0.6 \mathrm{um}$ 1P3M digital CMOS technology. The features of the proposed design include a loadoptimized 3-stage VCO, a frequency limiter RC circuit, and a ratioed VCO controlling current mirror. The jitter, thus, is reduced to $72.693 \mathrm{ps}$ at $600 \mathrm{MHz}$ at the presence of supply noise, while the sensitivity is limited to $286.6 \mathrm{ps} / \mathrm{V}$. This high-noise immunity design allows that the PLL can be integrated with digital circuits.
\end{abstract}

Keywords: Phase-locked loop; VCO; Low-jitter; 1P3M CMOS technology; Load-optimized; VLSI

\section{INTRODUCTION}

PLLs are often used in the $\mathrm{I} / \mathrm{O}$ interfaces of digital ICs in order to hide clock distribution delays and improve the overall system timing [5] or high frequency clock generators $[2,3,7]$. However, the noisy environment in which the ICs operate might introduce unwanted jitter from the expected timing at the output of the PLL. With a demand on high frequency and high speed PLLs, the design of low jitter PLLs thus become very challenging. Though many PLL designs have been proposed $[1,4,6,7]$, several design factors have long been ignored. First, the lock time of the PLL should be as short as possible. Second, the lowest output frequency might not necessarily be zero. Third, the size ratio of the conventional ring oscillator type VCO (voltage controlled oscillator) should be tuned and might be the same at every stage of inverters. In this work, we present a PLL design including a load-optimized 3-stage VCO, a frequency limiter RC circuit, and a ratioed VCO controlling current mirror. The targeted frequency is $500 \mathrm{MHz}$, while the output clock is ranged between $380 \mathrm{MHz}$ and

* This research was partially supported by National Science Council under grant NSC 87-2215-E-110-010.

${ }^{\dagger}$ Corresponding author. e-mail: ccwang@ee.nsysu.edu.tw 
$600 \mathrm{MHz}$. The fast lock time is 95 us in the worst case. The entire design is implemented by TSMC (Taiwan Semiconductor Manufacturing Company) 0.6 um IP3M CMOS technology and verified by HSPICE.

\section{VCO DESIGN FOR LOW-JITTER PLL}

As shown in Figure 1, the entire PLL circuits consists of a PFD (phase-freq detector), a charge pump, a second order loop filter, a VCO and a digital divider. In our design, the reference input clock is given $20 \mathrm{MHz}$ which can be easily produced by most of the commercial crystal oscillators.

\subsection{Frequency Limiter}

Most of the PLLs are designed specifically for certain pre-determined operating frequency. Thus there is no need to sacrifice the lock time for the working frequency range from $0 \mathrm{MHz}$ to a desired upper bound. Hence, we introduce a practical frequency limiter between the charge pump and the current mirror for ring oscillator in the VCO. The detailed frequency limiter is shown in Figure 2. We tend to take advantage of the $\mathrm{W} / \mathrm{L}$ ratio of NMOS M2 to clamp the output frequency range and the upper bound of this range such that the actually highest frequency of the PLL is not the same as that of the ring OSC. Besides, this highest output frequency of the output frequency range

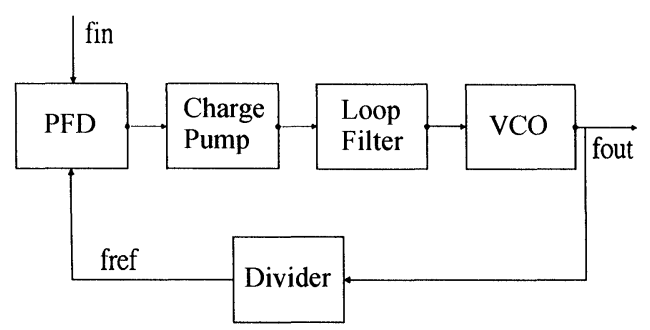

FIGURE 1 Block diagram of PLL. can be determined by adjusting the resistance of $R 1$ in Figure 2.

In contrast, M3 itself can be deemed as a nonlinear RC circuit which is able to control the lowest output operating frequency. The smaller the $\mathrm{W} / \mathrm{L}$ of M3, the lower the output frequency is. However, the ring OSC will not function if the W/L of M3 is smaller than a certain value. The size of M3 is thus used as an adjustable mechanism to determine the range of the output frequency. Another major benefit of such a circuit is to reduce the noise coupling effect of power supply to the ring OSC besides the mentioned frequency limiting function.

\subsection{Load-optimized Ring OSC}

Conventionally the ring OSC of the VCO used in high frequency PLLs is composed of three cascaded inverters with equal size. The might lead to a problem if the output of the VCO is connected to a buffer. It indicates that the last stage of the OSC possesses two loads while the other stages have only one. If the buffer size is sufficiently large in order to drive large load, the output of the VCO will damp to a stable voltage value if $V$ in of the frequency limiter is small. Hence, we adopt a ratioed design for the cascaded inverters to optimize the loading at each stage. The sizes of the transistors used in the OSC are shown in Figure 2.

\subsection{Fast Lock Time Consideration and Compensation}

The mentioned frequency limiter basically provides an almost pre-determined bias voltage at the input of the VCO. Referring to Figure 3, the $V$ - $f$ diagram of the proposed VCO reveals that our design strictly focuses the targeted range, $500 \mathrm{MHz}$. The lock time will be drastically reduced owing to the nonezero output frequency given the lowest input voltage to the VCO. However, the drawback of the narrow band effect can be compensated by the digital frequency divider shown in Figure 1. 


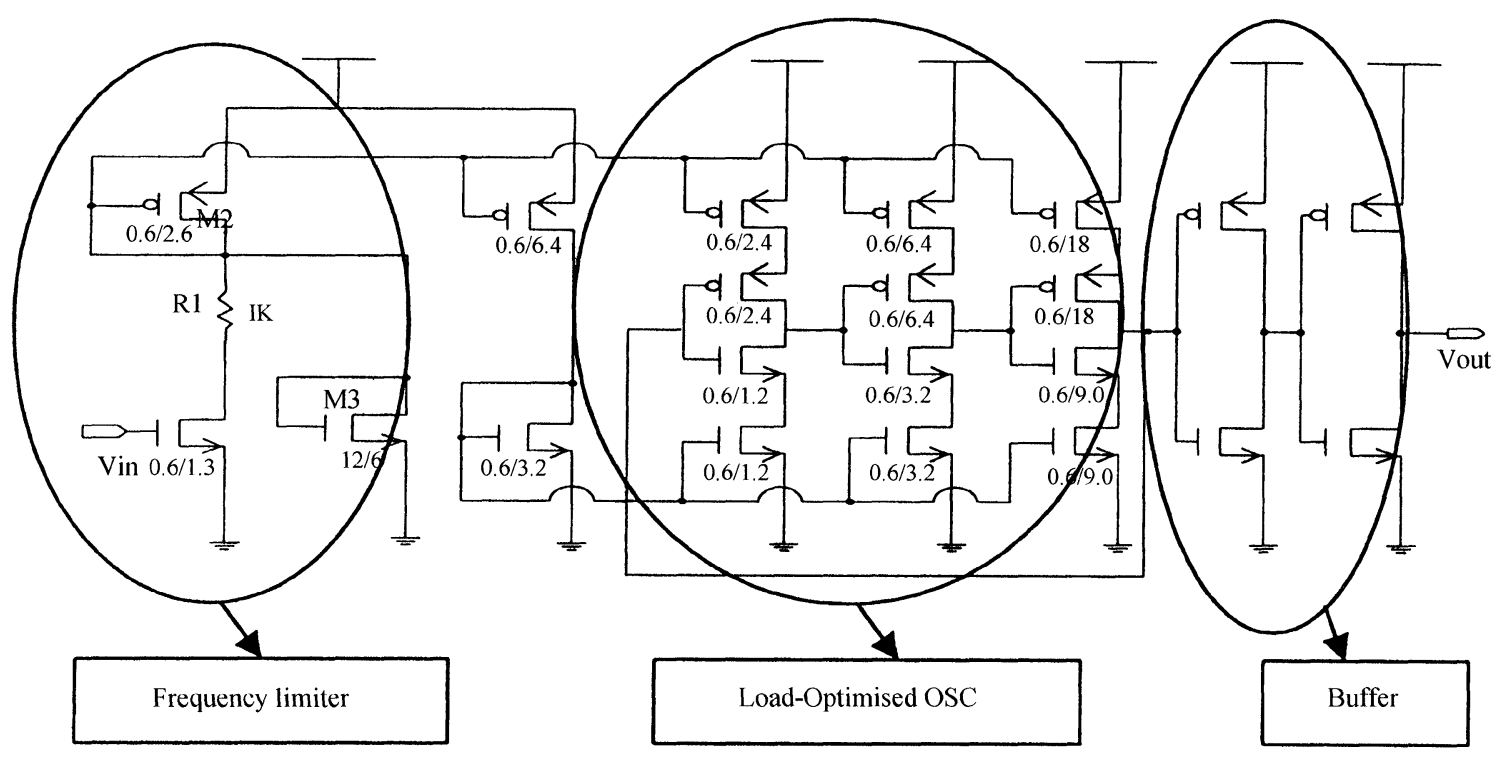

FIGURE 2 Schematic of the entire PLL.

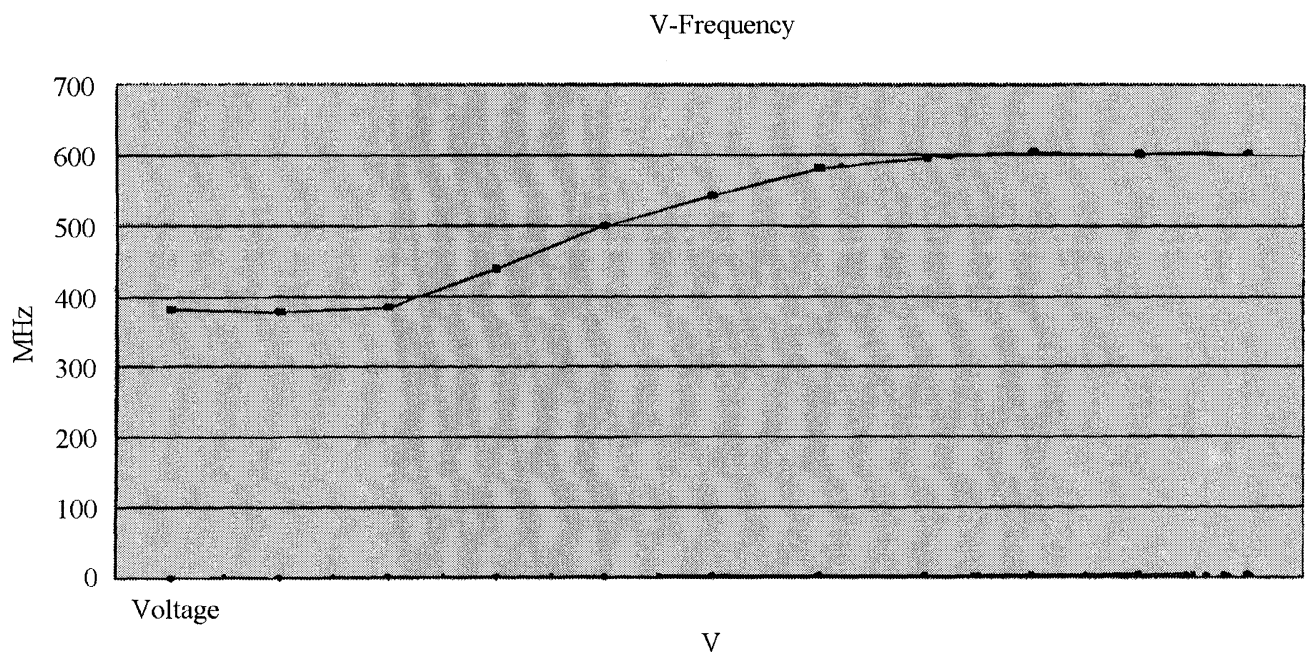

FIGURE 3 Schematic of the propose VCO.

\section{SIMULATION RESULTS AND CHIP LAYOUT}

To verify the correctness and functionality of the proposed design, we design the entire PLL circuit using TSMC 0.6 um 1P3M CMOS technology. The schematic and the layout are respectively shown in Figures 4 and 5. The capacitors in the loop filter are composed of gate-to-bulk capacitors of 50 parallel NMOS transistors, respectively. 


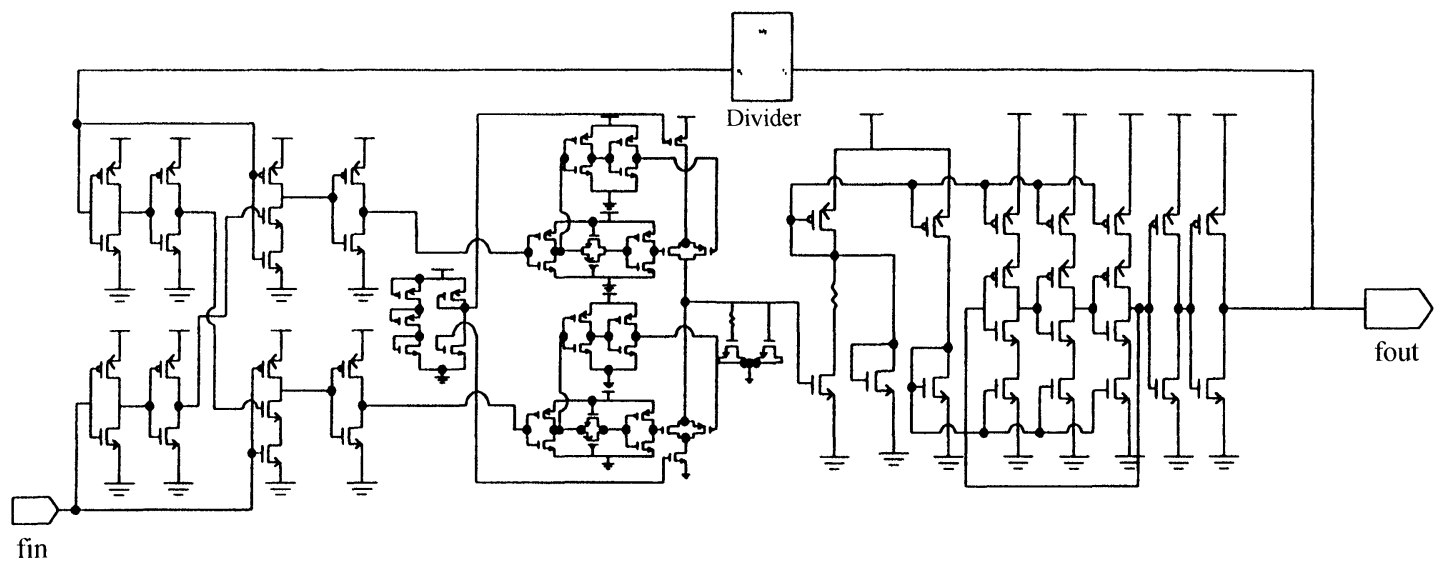

FIGURE 4 V-f diagram of VCO.

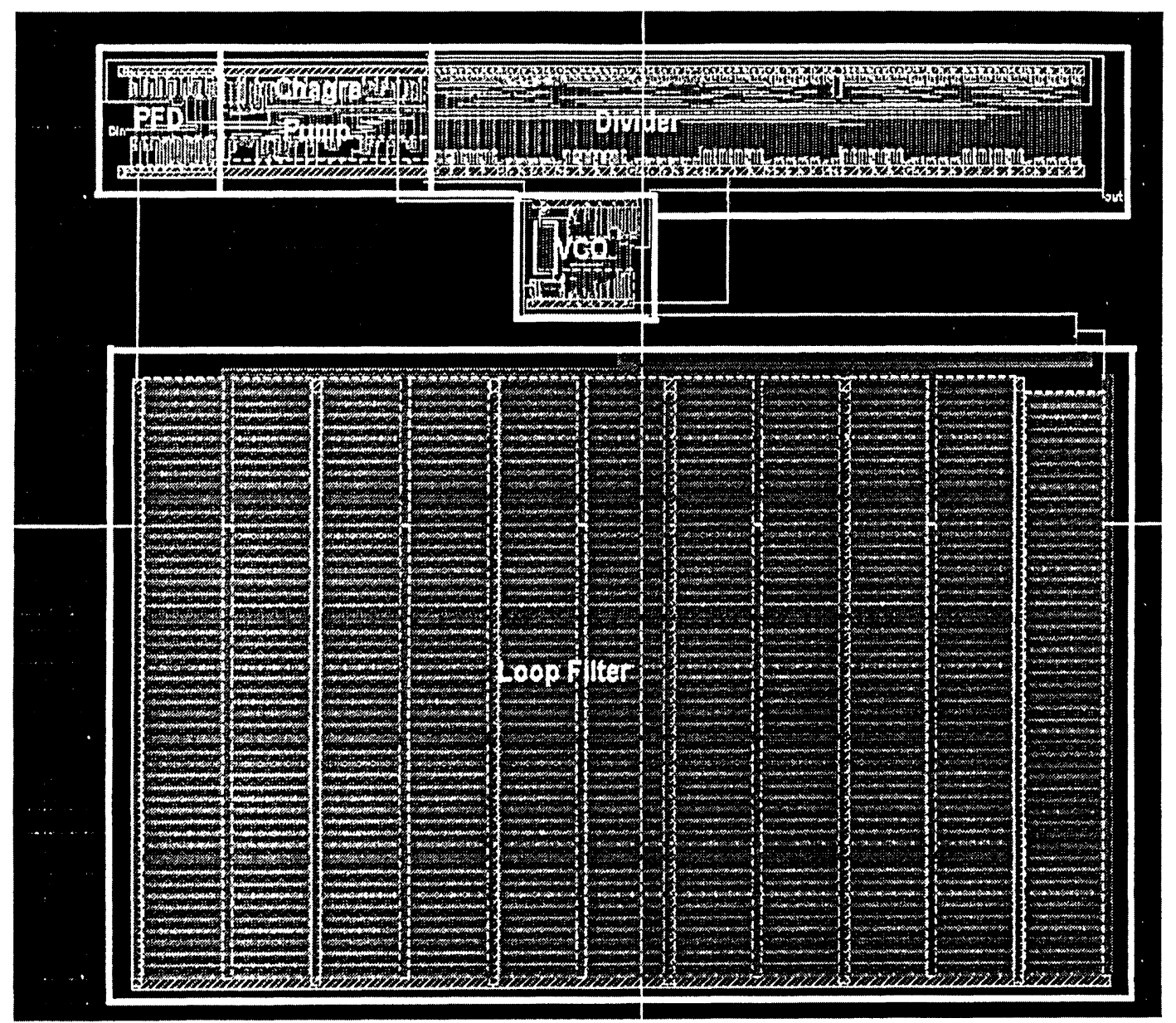

FIGURE 5 Gain-bandwidth of the loop filter. 


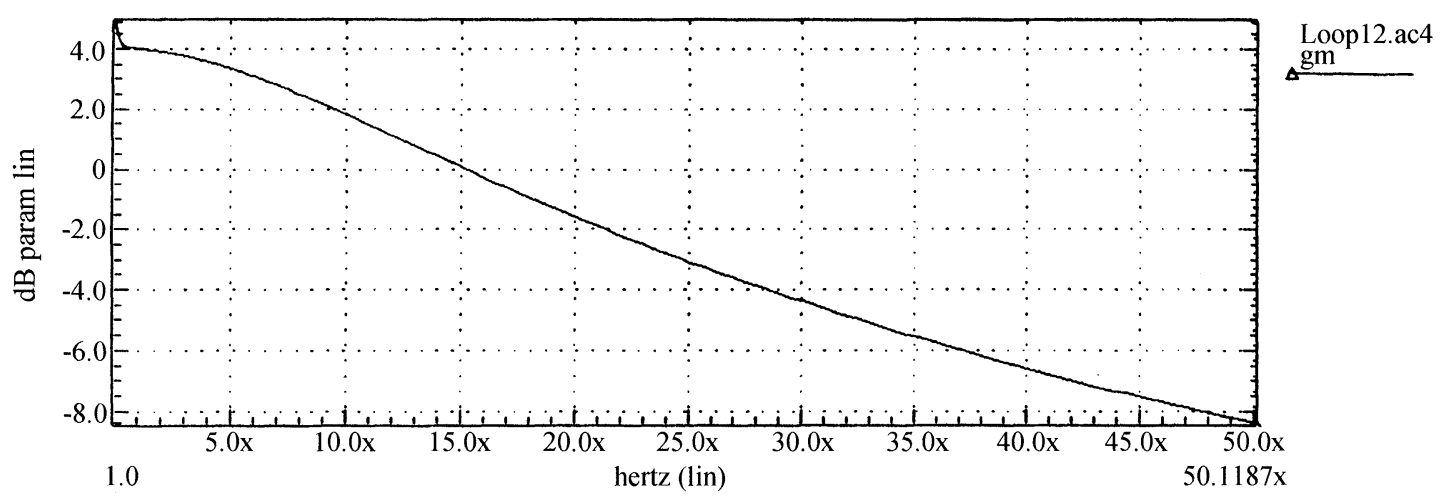

FIGURE 6 Layout of the propose PLL.

TABLE I Cycle-to-cycle VCO jitter at $600 \mathrm{MHz} @ 5 \mathrm{~V} 0.6$ um 1P3M CMOS

jitter without supply noise

jitter given noise of $0.5 \mathrm{~V} 1 \mathrm{MHz}$ square wave

Sensitivity $\pm 21.023 \mathrm{ps}$

$-70.6 /+72.693 \mathrm{ps}$

$286.6 \mathrm{ps} / \mathrm{V}$

TABLE II Jitter comparison without noise presence

\begin{tabular}{lcrll}
\hline & Lock time & Jitter & Lock freq. & Technology \\
\hline$[4]$ & N/A & $80 \mathrm{ps}$ & $250 \mathrm{MHz}$ & $0.5 \mathrm{um}$ CMOS 3V \\
{$[5]$} & $\mathrm{N} / \mathrm{A}$ & $144 \mathrm{ps}$ & $250 \mathrm{MHz}$ & $0.5 \mathrm{um}$ CMOS 3.3V \\
{$[8]$} & $\mathrm{N} / \mathrm{A}$ & $81 \mathrm{ps}$ & $130 \mathrm{MHz}$ & 0.8 um CMOS 5V \\
Ours & $43 \mathrm{u}$ & $160 \mathrm{ps}$ & $480 \mathrm{MHz}$ & 0.6 um CMOS 5V \\
\hline
\end{tabular}

TABLE III Jitter comparison with noise presence

\begin{tabular}{lcccccc}
\hline & $\begin{array}{c}\text { Jitter } \\
\mathrm{p}-\mathrm{p}(\mathrm{ps})\end{array}$ & $\begin{array}{c}\text { Std. } \\
(\mathrm{ps})\end{array}$ & $\begin{array}{c}\text { Noise- } \\
\text { level(v) }\end{array}$ & $\begin{array}{c}\text { Sensitivity } \\
\mathrm{ps} / \mathrm{V}\end{array}$ & $\begin{array}{c}\text { Freq. } \\
(\mathrm{MHz})\end{array}$ & Technology \\
\hline$[1]$ & 133 & 20.97 & 0.5 & $\mathrm{~N} / \mathrm{A}$ & 360 & 0.5 um CMOS 3.3V \\
Ours & $-70.6 /+72.7$ & 52.2 & 0.5 & 286.6 & 480 & 0.6 um CMOS 5V \\
\hline
\end{tabular}

That is, the source and drain of these transistors are all grounded. The gain-bandwidth diagram is given in Figure 6.

The measured sensitivity and the jitter performance is summarized in Table I.

The comparison of our design and prior works are also illustrated in the following Tables II and III.

Basing on the above results, the proposed design is superior to the prior works regarding the jitter and lock time.
TABLE IV Jitter measured by IMS200, which introduces about $0.2 \mathrm{~V}$ power noise

\begin{tabular}{lcc}
\hline Lock freq. (MHz) & Jitter $(\mathrm{ps})$ & Std. $(\mathrm{ps})$ \\
\hline 266.64 & 48 & 8.213 \\
300.00 & 54 & 9.076 \\
328.80 & 80 & 11.05 \\
360.96 & 111 & 16.84 \\
390.24 & 150 & 23.71 \\
420.96 & 100 & 15.40 \\
452.88 & 80 & 12.79 \\
466.08 & 70 & 2.110 \\
\hline
\end{tabular}




\section{REAL CHIP TESTING RESULT}

Ours design has been fabricated by TSMC and sponsored by CIC (Chip Implementation Center) of NSC (National Science Council). The chip no. is T06-88B-15. The testing equipment and environment are as follows: the power supply and the input clock are produced by ATM's
IMS200, which introduces about $0.2 \mathrm{~V}$ power noise. The output frequency is measured by a oscilloscope. The room temperature is about $24^{\circ} \mathrm{C}$. The total number of samples is 5,000. The real chip testing results are summarized in Tables IV and V, which respectively reveal the jitter measurement result and the operating frequency range.

TABLE V Frequency measured by IMS200, which introduces about $0.2 \mathrm{~V}$ power noise

\begin{tabular}{lcccc}
\hline Lock freq. (MHz) & Mean freq. (MHz) & Max freq. (MHz) & Min freq. (MHz) \\
\hline 266.64 & 266.5 & 268.2 & 264.8 \\
300.00 & 298.7 & 301.2 & 296.5 & 325.4 \\
328.80 & 329.2 & 334.1 & 354.1 & 0.590 \\
360.96 & 360.4 & 368.6 & 380.2 & 1.202 \\
390.24 & 391.1 & 403.2 & 412.5 & 2.185 \\
420.96 & 421.7 & 430.2 & 445.7 & 2.550 \\
452.88 & 453.6 & 462.1 & 458.6 \\
466.08 & 466.5 & 473.9 & & 2.501 \\
\hline
\end{tabular}

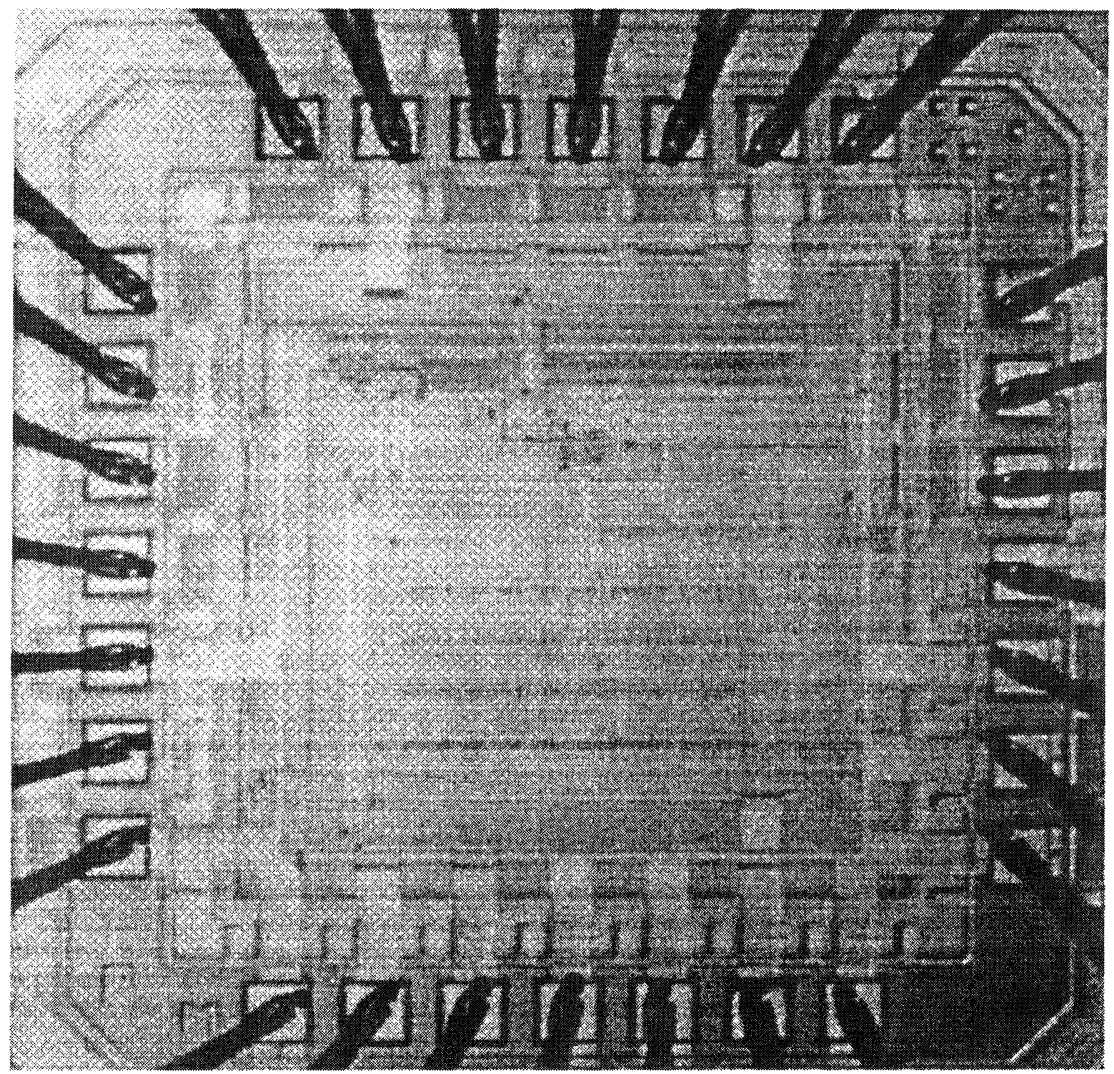

FIGURE 7 Die photo of our chip. 
The die photo of the chip is shown in Figure 7. The locked frequency range of our real chip is between $266 \mathrm{MHz}$ and $466 \mathrm{MHz}$ which is a little lower than what we expected in Section 2.3 $(500 \mathrm{MHz})$. The worse case of the jitter is $150 \mathrm{ps,}$ which is better than the simulation. There are two reasons why the performance of the real chip is better than the simulation. First, the significant digits of the simulation data are not long enough such that the precision is underestimated. Second, since the, frequency of the real chip is lower than the expectation value of the simulation results, a smaller jitter is then generated. Nevertheless, the real chip testing result is very close to the simulation results.

\section{CONCLUSION}

A novel and practical VCO design for digital PLL is presented in this paper. Besides shortening the lock time, the jitter caused by noise of the power supply is also reduced. Meanwhile, the loading effect of the ring OSC is taken into consideration. Simulation results provided by HSPICE reveal the value of the entire design. The performance of the real chip results show that our design is a practical approach.

\section{References}

[1] Zhang, Z.-X., Du, H. and Lee, M. S. (1996). "A $360 \mathrm{MHz}$ 3V CMOS PLL with $1 \mathrm{~V}$ peak-to-peak power supply noise tolerance", 1996 International Symp. On Solid-state Circuits, pp. 134-135.

[2] von Kaenel, V., Aebischer, D., van Dongen, R. and Piguet, C. (1998). "A $600 \mathrm{MHz}$ CMOS PLL microprocessor clock generator with a $1.2 \mathrm{GHz}$ VCO", 1998 International Symp. on Solid-state Circuits, pp. 320-323.

[3] Alvarez, J., Sanchez, H., Gerosa, G. and Countryman, R., "A wide-bandwidth low-voltage PLL for Power PC microprocessors", IEEE J. of Solid-state Circuits, SC-30, 383-391, April, 1995.

[4] von Kaenel, V., Aebischer, D., Piguet, C. and Dijkstra, E., "A $320 \mathrm{MHz}, 1.5 \mathrm{~mW} @ 1.35 \mathrm{~V}$ CMOS PLL for microprocessor clock generation", IEEE J. of Solid-state Circuits, 31(11), 1715-1722, November, 1996.
[5] Maneatis, J. G., "Low-jitter process-independent DLL and PLL based on self-biased techniques", IEEE $J$. of Solidstate Circuits, 31(11), 1723-1732, November, 1996.

[6] Johansson, H. O., "A simple precharged CMOS phase frequency detector", IEEE J. of Solid-state Circuits, 33(2), 295-299, February, 1998.

[7] Waizman, A. (1994). "A delay line loop for frequency synthesis of de-skewed clock", 1994 International Symp. on Solid-state Circuits, pp. 298-299.

[8] Yang, H. C., Lee, L. K. and Co, R. S., "A low jitter 0.3$165 \mathrm{MHz}$ CMOS PLL frequency synthesizer for $3 \mathrm{~V} / 5 \mathrm{~V}$ operation", IEEE J. of Solid-state Circuits, 32(4), 582-586, April, 1997.

[9] Baker, R. J., Li, H. W. and Boyce, D. E., "CMOS circuit design, layout, and simulation", Reading: IEEE Press, 1998.

\section{Authors' Biographies}

Chua-Chin Wang was born in Taiwan in 1962. He received the B.S. degree in electrical engineering from National Taiwan University in 1984, and the M.S. and Ph.D. degree in electrical engineering from State University of New York in Stony Brook in 1988 and 1992, respectively. $\mathrm{He}$ is currently a Professor in the Department of Electrical Engineering, National Sun Yat-Sen University, Kaohsiung, Taiwan. His recent research interests include low power and high speed logic circuit design, VLSI design, neural networks, and interfacing $\mathrm{I} / \mathrm{O}$ circuits.

Yu-Tsun Chien was born in Taiwan on December 4, 1974. He received the B.S. degree in electrical engineering and business administration from National Sun Yat-Sen University, Kaohsiung, Taiwan, in 1998. He is currently pursuing the M.S. degree in electrical engineering at National Sun Yat-Sen University. His current research interests are in high-speed analog integrated circuit design and IrDA transceivers.

Ying-Pei Chen was born in Taiwan on January 26, 1974. She received the B.S. degree in computer science information engineering from Tamkang University, Taipei, Taiwan, in 1998. She is currently pursuing the M.S. degree in electrical engineering at National Sun Yat-Sen University. Her current research interests are in networking and VLSI design. 

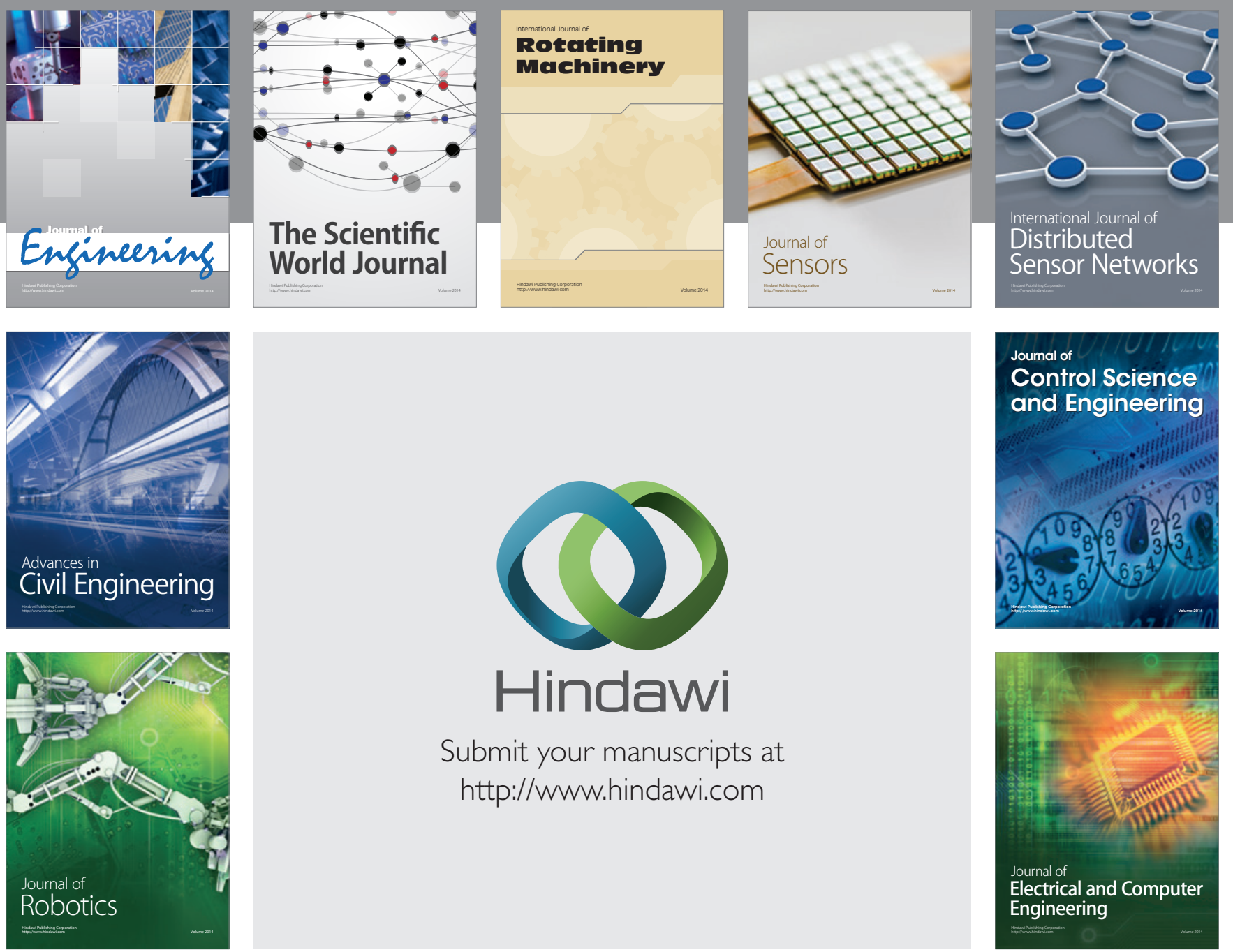

Submit your manuscripts at

http://www.hindawi.com
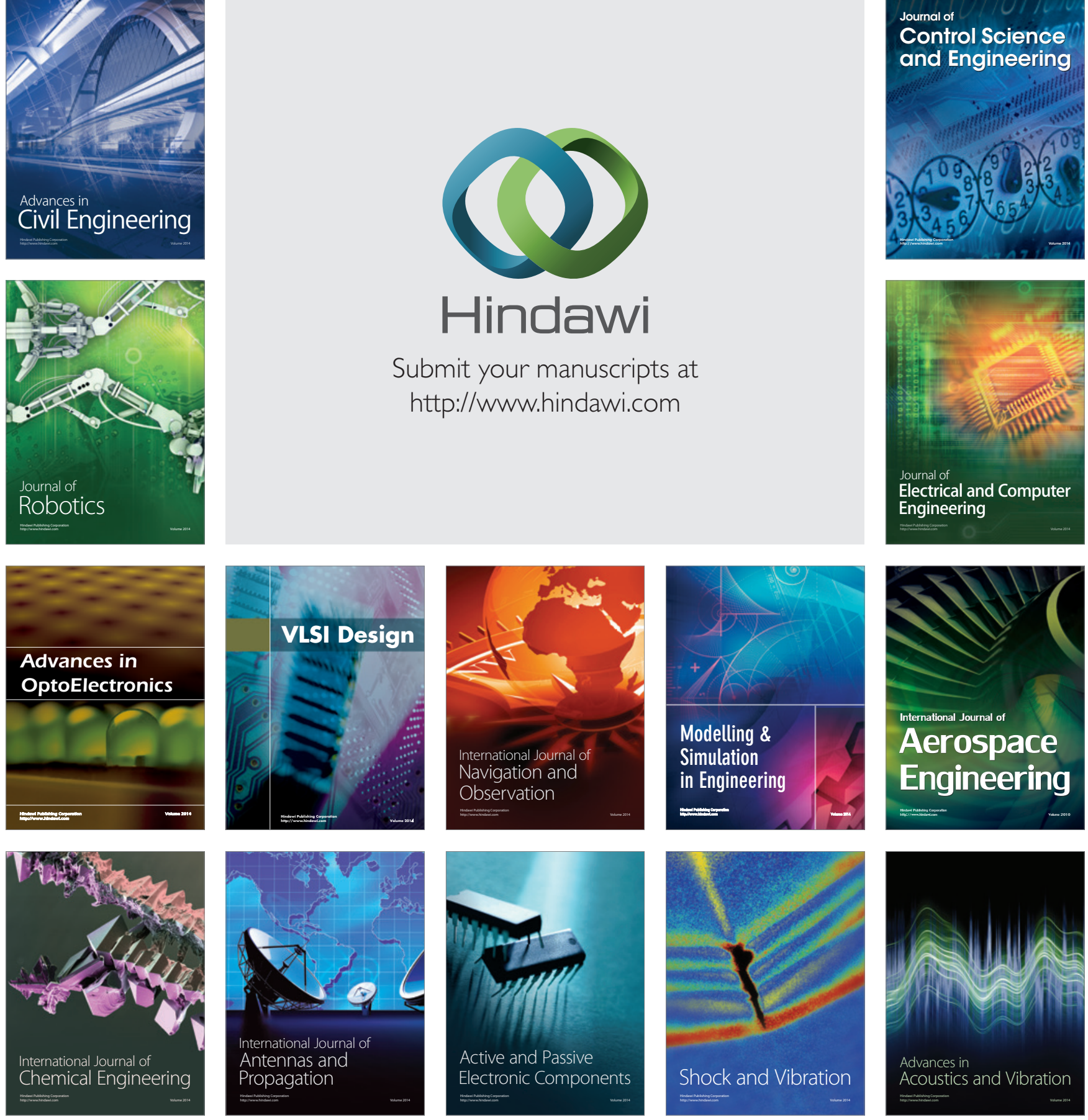